\title{
Unilateral and Bilateral Percutaneous Kyphoplasty for Thoracolumbar Osteoporotic Compression Fractures
}

\author{
Jin Tang1, Wei-chun Guo2, Jin-feng $\mathrm{Hu}^{2}$ and Ling $\mathrm{Yu}^{2}$ \\ ${ }^{1}$ Hubei 672 Orthopaedics Hospital of Integrated Chinese \& Western Medicine, Hongshan District, Wuhan Hubei, China \\ ${ }^{2}$ Department of Orthopedics, Renmin Hospital of Wuhan University, Wuhan, China
}

\begin{abstract}
Objective: To compare the efficacies of unilateral and bilateral percutaneous kyphoplasty (PKP) in the treatment of thoracolumbar osteoporotic compression fractures.

Study Design: Experimental study.

Place and Duration of Study: Department of Orthopedics, Hubei 672 Orthopedics Hospital of Integrated Chinese and Western Medicine, China, from November 2014 to January 2017.

Methodology: One hundred and seventy-eight patients with single-level thoracolumbar osteoporotic compression fractures included in this study. These patients were randomly assigned to unilateral $(n=83)$ and bilateral $(n=95)$ PKP groups. The operation time, bone cement volume, number of X-ray views, preoperative and postoperative pain visual analogue scale scores (VAS), Oswestry Dysfunction Index (ODI), Cobb angle, and vertebral height in both groups were recorded.

Results: Operation time, bone cement volume and intraoperative number of X-ray views were significantly different between unilateral and bilateral PKP groups $(29.8 \pm 2.7$ vs. $31.5 \pm 3.9$ minutes; $9.3 \pm 2.6$ vs. $11.2 \pm 3.7$ times; $3.1 \pm 0.7$ vs. $3.5 \pm 1.2 \mathrm{ml}$, respectively, $p<0.05$ ). VAS scores and ODI in both groups were significantly different before and 24 hours, 3 months and 6 months after surgery. The heights of anterior and middle borders of vertebral body and Cobb angle in the two groups were significantly different before and after surgery $(p<0.05)$.

Conclusion: The short-term efficacy was identical between unilateral and bilateral PKP. The unilateral PKP is characterized by short operation time, low hospital cost, less number of X-ray views and less bone cement volume. The unilateral PKP shows a higher risk of re-fracture of adjacent vertebral body compared with the bilateral PKP.
\end{abstract}

Key Words: Percutaneous kyphoplasty, Unilateral puncture, Bilateral puncture, Osteoporotic compression fractures, Visual analogue scale scores, Oswestry dysfunction index.

How to cite this article: Tang J, Guo WC, Hu JF, Yu L. Unilateral and bilateral percutaneous kyphoplasty for thoracolumbar osteoporotic compression fractures. J Coll Physicians Surg Pak 2019; 29(10):946-50.

\section{INTRODUCTION}

Osteoporosis is a metabolic bone disease characterised by bone loss and bone microstructure changes. The prevalence of postmenopausal osteoporosis is estimated to be $40-60 \%$ in women aged 70 years, and $60-75 \%$ in women aged more than 80 years old.1,2 Osteoporotic vertebral compression fractures (OVCFs) are the most common form of osteoporotic fractures, especially in elderly women over the age of 60 years, which are most common in the thoracolumbar spine (T11-L2). ${ }^{3}$

Conventional treatments include conservative treatment and open surgery. Conservative treatment includes bed

Correspondence to: Jin Tang, Hubei 672 Orthopaedics

Hospital of Integrated Chinese \& Western Medicine, No. 279

Luoyu Road, Hongshan District, Wuhan 430079, Hubei, China

E-mail: tangjin725@126.com

Received: March 14, 2019; Revised: June 14, 2019;

Accepted: July 09, 2019 rest, analgesia, brace protection and physical therapy. Usually, symptoms can be relieved in $2 / 3$ patients after 4 weeks of conservative treatment. ${ }^{4}$ However, approximately one-third patients still suffer from chronic intractable pain. ${ }^{5}$ Conservative treatment time is long, and the curative effect is uncertain. Long-term bedridden is easy to accompany complications. Thus, conservative treatment is often difficult to be accepted by patients and their families.

Conventional open surgery is traumatic and shows excessive bleeding. Because of osteoporosis, holding force of pedicle screw is not enough, which easily induces unstable fixation, leading to treatment failure. Therefore, elderly patients who do not have nerve compression symptoms are generally not recommended for internal fixation. Percutaneous kyphoplasty (PKP) is one of the most common methods for the treatment of OVCFs. PKP is minimally invasive, safe, quick to relieve pain and restore vertebral height.4,6-9 Previously, bilateral puncture is commonly used in PKP. Recently, 
unilateral PKP is increasingly recognised because of its short duration, low bone cement volume and low cost. 10

The objective of this study was to compare the efficacies of unilateral and bilateral PKP in the treatment of thoracolumbar osteoporotic compression fractures.

\section{METHODOLOGY}

This research was approved by the Medical Ethics Committee of the Second Hospital of Baoding City. Written informed consents were formally obtained from all participants. Between November 2014 to January 2017, 178 patients with single-level thoracolumbar osteoporotic compression fractures were recruited in this study. Patients were randomly assigned to unilateral $(n=83)$ and bilateral $(n=95)$ PKP groups.

Patients meeting all the following criteria were included: Patients aged $>65$ years, without history of trauma, lumbar fractures and lumbar surgery, with severe pain in the back and waist, limited motion, obvious percussion tenderness in the affected part, no obvious symptoms or signs of spinal cord or nerve compression, X-ray evidence T11-L2 single vertebral level compression degree between $25 \%$ and $75 \%$, preoperative CT scans demonstration intact posterior wall and pedicle, or dysplasia of vertebral pedicle in the injured vertebra, preoperative MRI showing high signal of the injured vertebra on fat-suppressed images, indicating fresh fractures; bone density with $\mathrm{T} \leq-2.5 \mathrm{SD}$, indicating definite osteoporosis; without any serious cardiovascular disease, or absolute surgical contraindication. Family members and patients agreed to surgery and patients had clear consciousness and could cooperate with postoperative rehabilitation and postoperative follow-up. The operation was performed by the same group of senior physicians using the same manufacturer's instruments. Patients were excluded if they had compression fractures of two or more vertebral bodies, previous history of lumbar fractures or lumbar spine surgery, other pathological fractures, incomplete burst fracture of posterior wall compression symptoms of the spinal cord or nerve or MRI revealed old fractures, refusal to operation, unconsciousness or mental illness, inability to cooperate with the treatment or postoperative recovery, or follow-up, inability to tolerate surgery or requiring other surgeries besides PKP.

Patients in both groups were kept in the prone position under local anesthesia. Chest and ilium were elevated with soft pillow. The abdomen was suspended. Before surgery, fluoroscopy was used to determine and mark projection of the injured vertebral body and bilateral pedicles. Local anesthesia with $1 \%$ lidocaine was applied to infiltrate the pedicle base. The needle was inserted at the outer upper margin of the pedicle. Approximately $0.5 \mathrm{~cm}$ transverse incision was made at the insertion point. When the abduction angle and the inclination angle of the guide needle were appropriate, the guide needle was rotated to the centre of the vertebral body under the supervision of the $\mathrm{C}$-arm machine. Along the guide needle, the working sleeve was screwed at approximately $1 \mathrm{~cm}$ anterior to the posterior border of the vertebral body. After removal of the guide needle, the hole was expanded with a drill at $0.5 \mathrm{~cm}$ posterior to the anterior border of the vertebral body. After balloon implantation, contrast medium was slowly injected to dilate the balloon. Balloon expansion and fracture reduction were observed under the $\mathrm{C}$-arm machine. When the balloon reached the endplate or the height of the vertebral body on this side was satisfactory, the balloon was removed. Bone cement in the drawing period was slowly injected into the vertebral body. The distribution of bone cement in the vertebral body was observed to avoid bone cement outleakage. The injection was terminated immediately after the discovery of leakage. When bone cement reached the posterior wall of the vertebral body, the injection was stopped. When the bone cement was solidified, the sleeve was removed. After suture, the incision was sterilely dressed. In the unilateral PKP group, puncture was performed at 10 o'clock position of the left pedicle; if there was a coronal deformity, the puncture was performed from the concave pedicle. In the bilateral PKP group, the puncture was conducted simultaneously at the 10 o'clock position of the left pedicle and the 2 o'clock position of the right pedicle. One side of the balloon was expanded, and then the other side was expanded by the same method. Subsequently, bone cement was injected simultaneously on both sides.

All patients in both groups were bedridden for 24 hours after surgery. Anti-infection, acid preparation and stomach protection, calcium and calcitriol were administered on the day of operation. Vital signs, motor sensation of lower extremities, urination and defecation were observed. After 24 hours of the operation, patients could wear waist brace and walk out of bed under the permissible condition. Simultaneously, oral alendronate sodium (or intramuscular salmon calcitonin) was administered. After hospitalisation for 3-5 days, the patients left the hospital. The patients continued to receive anti-osteoporosis treatment after discharge.

Operation time, bone cement volume, number of X-ray views, preoperative and postoperative pain visual analogue scale (VAS) scores, preoperative and postoperative Oswestry Dysfunction Index (ODI), preoperative and postoperative Cobb angle, vertebral height, and postoperative complications were observed and compared in both groups. Measurement data were expressed as the mean \pm SD. VAS scores, ODI, the height of vertebral anterior border, height of vertebral middle part, and Cobb angle were compared using $t$ test. Count data were compared using Chi-squared test. All data were analysed with SPSS version 19.0 software. Statistical significance was defined at $p<0.05$. 


\section{RESULTS}

Unilateral PKP group ( $\mathrm{n}=83$ ) contained 26 males and 57 females, at the age of 68-92 years (mean $72.3 \pm 3.6$ years). Injured positions were T11 in 9 cases, T12 in 32 cases, L1 in 27 cases, and L2 in 15 cases. Injury time was $1-12$ days (mean $3.5 \pm 1.2$ days). Follow-up time was $7-15$ months (mean $9.3 \pm 1.8$ months). Bilateral PKP group $(n=95)$ contained 32 males and 63 females, at the age of $70-88$ years (mean $73.9 \pm 2.8$ years). Injured positions were T11 in 7 cases, T12 in 37 cases, L1 in 33 cases, and L2 in 18 cases. Injury time was 1-15 days (mean $4.2 \pm 2.6$ days). Follow-up time was 7-13 months (mean $8.5 \pm 1.6$ months). No significant difference in general data was detected between the two groups ( $p>0.05$ ), with comparability. Surgery was successfully completed in 178 patients in both groups. There was no obvious bone cement reaction during surgery. No pulmonary embolism, bone cement leakage or nerve injury occurred during and after surgery. No infection appeared after surgery.

The mean operation time was $29.8 \pm 2.7$ minutes; intraoperative mean number of X-ray views was $9.3 \pm 2.6$ times; mean bone cement volume was $3.1 \pm 0.7 \mathrm{ml}$ in the unilateral PKP group. Mean operation time was 31.5 \pm 3.9 minutes; intraoperative mean number of $\mathrm{X}$-ray

Table I: Comparison of VAS scores and ODI in both groups before and after surgery.

\begin{tabular}{l|c|c|c|c}
\hline & $\begin{array}{c}\text { Unilateral } \\
\text { PKP group }\end{array}$ & $\begin{array}{c}\text { Bilateral } \\
\text { PKP group }\end{array}$ & t-value & $\mathrm{P}$ \\
\hline VAS scores & $7.9 \pm 1.2$ & $7.8 \pm 1.4$ & 0.508 & 0.612 \\
Preoperative & $3.5 \pm 1.4$ & $3.2 \pm 1.0$ & 1.660 & 0.099 \\
Postoperative 24 hours & $2.8 \pm 0.7$ & $2.7 \pm 0.6$ & 1.026 & 0.306 \\
Postoperative 3 months & $2.7 \pm 0.8$ & $2.6 \pm 0.4$ & 1.075 & 0.284 \\
Postoperative 6 months & & & & \\
\hline ODI & $87.3 \pm 7.2$ & $86.4 \pm 6.3$ & 0.889 & 0.375 \\
Preoperative & $26.5 \pm 2.9$ & $25.8 \pm 2.0$ & 1.893 & 0.06 \\
Postoperative 24 hours & $24.1 \pm 2.3$ & $23.8 \pm 1.9$ & 0.953 & 0.342 \\
Postoperative 3 months & $23.5 \pm 2.5$ & $22.9 \pm 1.8$ & 1.853 & 0.065 \\
Postoperative 6 months & 23.5 &
\end{tabular}

views was $11.2 \pm 3.7$ times; mean bone cement volume was $3.5 \pm 1.2 \mathrm{ml}$ in the bilateral PKP group. Operation time and intraoperative number of $X$-ray views were significantly different between the two groups $(p<0.01)$. Bone cement volume were different between the two groups $(p=0.008)$. VAS scores and ODI in both groups were significantly different before and after surgery (Table I). The heights of anterior and middle borders of vertebral body and Cobb angle in the two groups were significantly different before and after surgery $(p<0.05$; Table II).

In the unilateral PKP group, five patients (6.02\%) affected re-fracture of adjacent vertebral bodies, which were recovered after the second PKP. In the bilateral PKP group, seven patients $(7.37 \%)$ suffered from refracture of adjacent vertebral bodies; one patient and his family members refused to receive surgery; the remaining six patients underwent the second PKP and the symptom recovered. The risk of re-fracture of adjacent vertebral body was higher in the bilateral PKP group compared with the unilateral PKP group.

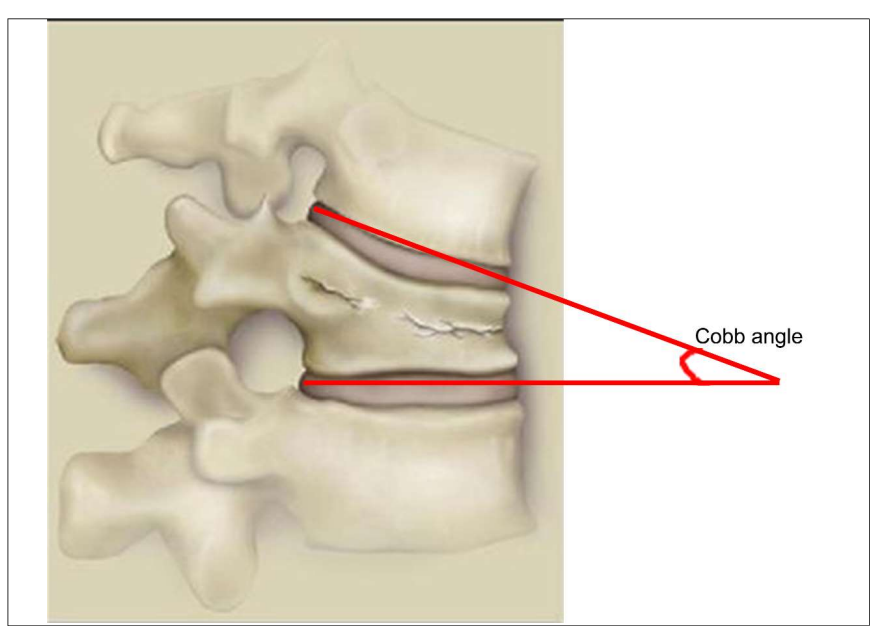

Figure 1: Cobb angle diagram. Cobb angle: The angle between the line of anterior and posterior borders of superior endplate and the line of anterior and posterior borders of inferior endplate of injured vertebra.

Table II: Comparison of the height of injured vertebra and Cobb angle in both groups before and after surgery.

\begin{tabular}{|c|c|c|c|c|}
\hline & $\begin{array}{l}\text { Unilateral } \\
\text { PKP group }\end{array}$ & $\begin{array}{l}\text { Bilateral } \\
\text { PKP group }\end{array}$ & t-value & $P$ \\
\hline \multicolumn{5}{|c|}{ Height of anterior border of injured vertebra (mm) } \\
\hline Preoperative & $16.4 \pm 1.8$ & $15.9 \pm 1.7$ & 1.905 & 0.058 \\
\hline Postoperative 24 hours & $23.6 \pm 2.1$ & $24.2 \pm 2.3$ & 1.808 & 0.072 \\
\hline Postoperative 3 months & $23.9 \pm 2.2$ & $24.5 \pm 2.1$ & 1.860 & 0.065 \\
\hline Postoperative 6 months & $23.8 \pm 2.3$ & $24.4 \pm 2.5$ & 1.658 & 0.099 \\
\hline \multicolumn{5}{|c|}{ Height of middle part of injured vertebra $(\mathrm{mm})$} \\
\hline Preoperative & $15.3 \pm 1.4$ & $15.7 \pm 1.6$ & 1.763 & 0.08 \\
\hline Postoperative 24 hours & $23.3 \pm 2.2$ & $23.7 \pm 2.3$ & 1.181 & 0.239 \\
\hline Postoperative 3 months & $23.8 \pm 2.5$ & $24.5 \pm 2.7$ & 1.786 & 0.076 \\
\hline Postoperative 6 months & $23.6 \pm 2.4$ & $24.3 \pm 2.6$ & 1.857 & 0.065 \\
\hline \multicolumn{5}{|l|}{ Cobb angle $\left({ }^{\circ}\right)$} \\
\hline Preoperative & $34.3 \pm 3.5$ & $33.8 \pm 3.4$ & 0.965 & 0.336 \\
\hline Postoperative 24 hours & $23.5 \pm 2.8$ & $22.8 \pm 2.6$ & 1.729 & 0.086 \\
\hline Postoperative 3 months & $23.1 \pm 2.4$ & $22.4 \pm 2.5$ & 1.899 & 0.059 \\
\hline Postoperative 6 months & $23.4 \pm 2.9$ & $22.6 \pm 2.7$ & 1.905 & 0.058 \\
\hline
\end{tabular}




\section{DISCUSSION}

In recent years, OVCFs have become more and more common in the clinic. In USA, the medical cost of OVCFs was $\$ 16.9$ billion in 2005 , and would increase to $\$ 25.3$ billion in 2025.11 In China, the incidence of osteoporosis was $70 \%$ in women aged $60-69$ years old, and approximately $30 \%$ in men. Twenty percent of osteoporosis patients over the age of 70 years suffered from varying degrees of OVCFs, and the mortality rate within 5 years was $23 \%-34 \% .{ }^{12}$

The emergence of PKP provides a safe and effective minimally invasive approach for OVCFs patients, can reduce the collapsed vertebral body after balloon dilation, form a complete lacuna and bone wall in the vertebral body, reduce the injection pressure of bone cement, immediately relieve pain, restore the vertebral height, and remarkably decrease leakage rate of bone cement. ${ }^{13}$ PKP is becoming more and more popular among doctors and patients. In our study, significant differences in VAS scores and ODI in both groups were determined before and after surgery; no significant differences in VAS scores and ODI before and after surgery were detected between the two groups. These findings suggested that unilateral and bilateral PKP could effectively mitigate pain, and no significant difference was measured between them. The principle that PKP can immediately relieve pain may lie in either injection of bone cement restoring the stability and biomechanical strength of the injured vertebra and reduce the stimulation of micro-motion after fracture to the vertebral nerve14; or the thermal and cytotoxic effects of cement polymerisation destroying the nerve endings and decreasing the sensitivity of nerve endings.

The standard PKP uses bilateral pedicle punctures and double balloons. Bilateral puncture can make the bone cement distribute evenly and symmetrically in the vertebral body. However, it increases the number of X-ray views, operation time, and operation cost. In our study, operation time, the number of X-ray views and bone cement volume were obviously higher in the bilateral PKP group than in the unilateral PKP group. Nevertheless, no significant difference in preoperative and postoperative height of the injured vertebra and Cobb angle was detected between the two groups. These results indicated that the unilateral puncture was equivalent to the bilateral puncture for the improvement of the clinical symptoms and the vertebral body height in OVCFs patients, but the risk of vertebral re-fracture increased in unilateral PKP group. Ioannis et al. found that no significant differences were determined in clinical efficacy and imaging between unilateral and bilateral PKP in 69 OVCFs patients. ${ }^{15} \mathrm{Li}$ et al. believed that bilateral PKP could improve Cobb angle of the coronal scoliosis, and it was strongly associated with the improvement of the quality of life of the patients after surgery. ${ }^{16}$ Chen et al. considered that unipedicular and bipedicular kyphoplasty could obviously increase biomechanical strength of the injured vertebra; 17 bone cement evenly distributed at both sides of the vertebra after bipedicular kyphoplasty, but at the puncture side after unipedicular kyphoplasty, which could induce uneven distribution of bone cement in the vertebral body, and significantly increased the possibility of re-fracture. Therefore, currently, the choice of PKP approach is controversial.

In this study, the unilateral PKP group achieved similar effects as the bilateral PKP group, and the recovery of vertebral height and Cobb angle was not significantly different from that of the bilateral PKP group, but the follow-up time was not enough, and the long-term effect cannot be compared. Combined with the experience, follow-up results and related literatures, the authors believed that under permitting conditions of patient's physical condition and cost, bilateral puncture was chosen as much as possible for severe compression. Unilateral puncture is mainly applicable to: patients with poor body condition, combined with heart and lung diseases, and cannot tolerate prolonged surgery; affordability issue; obvious osteoporosis, when appropriately increased abduction angle by using unilateral puncture can achieve the same effects as bilateral puncture; vertebral compression or collapse is confined to one side; and multilevel vertebral compression fracture.

\section{CONCLUSION}

Unilateral and bilateral PKP are safe and effective minimally invasive methods for treating OVCFs. The short-term effect is quite the same between unilateral and bilateral PKP. Unilateral PKP is characterised by short operation time, low cost, less number of X-ray views, and less bone cement volume. Nevertheless, the unilateral PKP shows a higher risk for re-fracture of adjacent vertebral body compared with the bilateral PKP. Under permitting conditions of patient's physical condition and cost, bilateral puncture was chosen as much as possible for severe compression. Due to the insufficient follow-up time, the long-term effect of the two methods should be supported by further follow-up.

\section{ETHICAL APPROVAL:}

This research was approved by Hubei 672 Orthopaedics Hospital of Integrated Chinese \& Western Medicine Ethics Committee.

\section{PATIENTS' CONSENT:}

Written informed consents were formally obtained from all participants.

\section{CONFLICT OF INTEREST:}

The authors report no conflict of interest. 


\section{AUTHORS' CONTRIBUTION:}

JT: Conceived the idea, conducted the analyses, provided the data, written and revised.

WCG: Conceived the idea, written and revised.

JFH: Conducted the analyses, provided the data, written and revised.

LY: Provided the data; written and revised.

\section{REFERENCES}

1. Patil S, Rawall S, Singh D, Mohan K, Nagad P, Shial B, et al. Surgical patterns in osteoporotic vertebral compression fractures. Eur Spine J 2013; 22:883-91.

2. Kanayama M, Oha F, Iwata A, Hashimoto T. Does balloon kyphoplasty improve the global spinal alignment in osteoporotic vertebral fracture? Int Orthop 2015; 39:1137-43.

3. Ge Z, Ma R, Chen Z, Zhang H, Ding H, Liang S, Suo Z. Uniextrapedicular kyphoplasty for the treatment of thoracic osteoporotic vertebral fractures. Orthopedics 2013; 36:e1020-4.

4. Yan L, Jiang R, He B, Liu T, Hao D. A comparison between unilateral transverse process-pedicle and bilateral puncture techniques in percutaneous kyphoplasty. Spine (Phila Pa 1976) 2014; 39:B19-26.

5. Venmans A, Klazen CA, Lohle PN, Mali WP, van Rooij WJ. Natural history of pain in patients with conservatively treated osteoporotic vertebral compression fractures: Results from VERTOS II. AJNR Am J Neuroradiol 2012; 33:519-21.

6. Yang H, Liu T, Zhou J, Meng B, Wang G, Zhu X. Kyphoplasty versus vertebroplasty for painful osteoporotic vertebral compression fractures - which one is better? A systematic review and meta-analysis. Int J Spine Surg 2013; 7:e45-57.

7. Xing D, Ma JX, Ma XL, Wang J, Xu WG, Chen Y, et al. A metaanalysis of balloon kyphoplasty compared to percutaneous vertebroplasty for treating osteoporotic vertebral compression fractures. J Clin Neurosci 2013; 20:795-803.

8. Chang X, Lv YF, Chen B, Li HY, Han XB, Yang K, et al. Vertebroplasty versus kyphoplasty in osteoporotic vertebral compression fracture: A meta-analysis of prospective comparative studies. Int Orthop 2015; 39:491-500.

9. Gu CN, Brinjikji W, Evans AJ, Murad MH, Kallmes DF. Outcomes of vertebroplasty compared with kyphoplasty: A systematic review and meta-analysis. J Neurointerv Surg 2016; 8:636-42.

10. Wang Z, Wang G, Yang H. Comparison of unilateral versus bilateral balloon kyphoplasty for the treatment of osteoporotic vertebral compression fractures. J Clin Neurosci 2012; 19:723-6.

11. Chen $\mathrm{H}$, Tang $\mathrm{P}$, Zhao $\mathrm{Y}$, Gao $\mathrm{Y}$, Wang $\mathrm{Y}$. Unilateral versus bilateral balloon kyphoplasty in the treatment of osteoporotic vertebral compression fractures. Orthopedics 2014; 37: e828-35.

12. Qiu GX. Treatment strategy of osteoporotic fracture in the elderly. Zhonghua Laonian Guke yu Kangfu Dianzizazhi 2015; 1:1-5.

13. Anderson PA, Froyshteter AB, Tontz WL Jr. Meta-analysis of vertebral augmentation compared with conservative treatment for osteoporotic spinal fractures. J Bone Miner Res 2013; 28: 372-82.

14. Lieberman IH, Dudeney S, Reinhardt MK. Initial outcome and efficacy of "kyphoplasty" in the treatment of painful osteoporotic vertebral compression fractures. Spine 2001; 26:1631-8.

15. Papanastassiou ID, Eleraky M, Murtagh R, Kokkalis ZT, Gerochristou M, Vrionis FD. Comparison of unilateral versus bilateral kyphoplasty in multiple myeloma patients and the importance of preoperative planning. Asian Spine J 2014; 8: 244-52.

16. Li QW, Wang YX, Zhang X, Meng CY. Effect of surgical area of cross-sectional scoliosis Cobb angle between unilateral and bilateral PKP for osteoporotic vertebral compression fractures. Zhonghua Linchuan Yishi Zazhi Dianziban 2016; 10:3365-9.

17. Chen B, Li Y, Xie D, Yang X, Zheng Z. Comparison of unipedicular and bipedicular kyphoplasty on the stiffness and biomechanical balance of compression fractured vertebrae. Eur Spine J 2011; 20:1272-80. 